\title{
Artigos
}

\section{Repercussões das diretrizes curriculares nacionais de 2014 nos projetos pedagógicos das novas escolas médicas}

\author{
Repercussions of the 2014 national curricular guidelines on the pedagogical \\ projects of new medical schools (abstract: p. 15)
}

Repercusiones de las directrices curriculares nacionales de 2014 en los Proyectos Pedagógicos de las nuevas escuelas médicas (resumen: p. 15)

\author{
Carolina Machado ${ }^{(a)}$ \\ <carolmachado@univali.br> \\ José Maurício de Oliveira(b) \\ $<$ jose.maoliveira@hsl.org.br> \\ Edson Malvezzi(c) \\ <edsonmalvezzi@yahoo.com.br>
}

\author{
(a) Curso de Medicina, \\ Universidade do Vale do Itajaí. \\ Rua Uruguai 458. Itajaí, SC, \\ Brasil. 88302-901. \\ (b) Instituto Sírio-Libanês de \\ Ensino e Pesquisa, Hospital \\ Sírio-Libanês. São Paulo, \\ SP, Brasil. \\ (c) Programa de Pós-Graduação \\ em Gestão da Clínica, \\ Universidade Federal de São \\ Carlos. São Carlos, SP, Brasil.
}

As Diretrizes Curriculares Nacionais orientam a constituição dos currículos para ordenamento da formação profissional em saúde e os projetos pedagógicos dos cursos as articulam às práticas pedagógicas. Esta pesquisa analisou as repercussões das Diretrizes Curriculares de 2014 nos projetos pedagógicos das escolas médicas criadas após sua publicação. De abordagem qualitativa, faz análise documental dos referidos projetos orientada pela hermenêutica crítica. Os achados revelam forte alinhamento dos projetos analisados às diretrizes curriculares. Identificou-se deslocamento do paradigma flexneriano para a integralidade, tendendo para formação de profissionais voltada às reais necessidades da população. Outros achados são as diferenças na concepção do médico de formação geral e na utilização das metodologias ativas de ensino-aprendizagem. Evidenciou-se ainda matrizes curriculares com concepções cuja proposta de integração ensino-serviço-comunidade não pareceu estar garantida, sugerindo a manutenção de propostas baseadas em disciplinas.

Palavras-chave: Educação médica. Currículo. Educação de graduação em Medicina. Política de educação superior. 


\section{Introdução}

Em 1910, Abraam Flexner publicou um estudo sobre escolas médicas nos EUA e no Canadá que produziu recomendaçôes para a formação desses profissionais, com destaque para a criação de hospitais-escola e um ensino pautado por uma prática científica positivista. Esse modelo reorientou o ensino médico em todo mundo e é considerado por muitos autores como um paradigma que ainda tem influenciado a formação em saúde $e^{1-3}$.

$\mathrm{Na}$ década de 1950, fóruns nacionais e internacionais começam a apontar a insuficiência deste modelo para balizar a formação médica, devido ao distanciamento dos currículos das necessidades de saúde da população $0^{4,5}$.

Destes fóruns, e no bojo da reforma sanitária brasileira, nasce um movimento para aproximar a formação dos profissionais de saúde às reais necessidades da população. Esse movimento é baseado na concepção da integralidade da atenção à saúde ${ }^{6,7}$.

A partir de 2001, com a publicação das Diretrizes Curriculares Nacionais (DCN) para os cursos de Medicina, o Ministério da Saúde assume seu papel indutor na formação de recursos humanos, até então liderado pelo Ministério da Educação. Um marco importante para fortalecer o papel do Ministério da Saúde foi a criação da Secretaria de Gestão do Trabalho e da Educação em Saúde (SGTES) ${ }^{8,9}$.

Em 2002, em parceria, os Ministérios da Saúde e da Educação criaram o Programa de Incentivo às Mudanças Curriculares dos Cursos de Medicina (Promed), que estimulava essas escolas a adequarem seus currículos às DCN de 2001. Inspirado no Promed, em 2005, foi criado o Programa Nacional de Reorientação da Formação Profissional em Saúde (Pró-Saúde), que expandiu o incentivo para os outros cursos da Saúde $e^{9-11}$.

Em 2008, por meio de nova portaria interministerial, foi instituído o Programa de Educação pelo Trabalho para a Saúde (PET-Saúde), com o objetivo de fortalecer a integração ensino-serviço ${ }^{12}$.

Outro marco importante foi a criação do Programa Mais Médicos em 2013, que, entre outras açôes, estabeleceu novos parâmetros para a formação médica no país e desencadeou a elaboração das novas DCN de Medicina que foram publicadas em 2014 $4^{13-15}$.

As DCN de 2014 têm a intenção de mudar os paradigmas dos cursos de medicina no Brasil, visando à formação de profissionais mais preparados para atuar no Sistema Único de Saúde (SUS) e por outro lado induzir as instituições de ensino superior (IES) a assumir responsabilidade social com o sistema público de saúde ${ }^{16}$. O aprendizado voltado para a integralidade do cuidado e a ênfase na Atenção Primária a Saúde (APS) são eixos importantes destas diretrizes ${ }^{17}$. No campo das concepçôes pedagógicas destacam-se o trabalho colaborativo em equipe de saúde com abordagem multidisciplinar, bem como a adoção de metodologias de ensino inovadoras ${ }^{16}$.

Desde então, estudos vêm sendo realizados sobre seus efeitos práticos ${ }^{16,18-20}$ e sobre sua própria concepção ${ }^{17,21,22}$, mas poucos se referem às repercussões nos projetos pedagógicos dos cursos (PPC) nas novas escolas médicas tal como aqui proposto. 


\section{Percurso metodológico}

Este estudo de abordagem qualitativa, com levantamento documental, tem como objetivo analisar as repercussóes das Diretrizes Curriculares Nacionais do curso de graduação em Medicina de 2014 nos PPC das novas escolas médicas criadas após sua publicação em 20 de junho de $2014^{14}$.

Desde então até o início da coleta de dados deste estudo, em fevereiro de 2017, foram criadas 53 escolas médicas. Para obtenção dos PPC, adotou-se a estratégia sequencial de acesso ao sítio eletrônico do Ministério da Educação (MEC), aos sítios eletrônicos das próprias escolas e, por último, para aquelas que não disponibilizavam, foi realizado contato formal por meio de mensagem para o endereço eletrônico do coordenador do curso, obtido no site do MEC. Ainda que sejam considerados documentos públicos, este processo resultou em uma amostra de $15 \mathrm{PPC}$, que foram incluídos neste estudo e identificados aleatoriamente de "E1" a "E15".

A sistematização do material coletado seguiu os referenciais metodológicos da análise de conteúdo, com ênfase na modalidade da análise temática ${ }^{23,24}$. Para tanto, foram utilizadas como categorias empíricas: produção do cuidado, gestão em saúde e conceitos pedagógicos. Optou-se por apresentar os resultados nas três dimensóes do perfil de competência das DCN de 2014.

A discussão se orienta por postura hermenêutica crítica, que busca evitar tornar o pesquisador surdo para outras e diversas "verdades", deixando-se dialogar e se interrogar pelo material em estudo, mais por seu estranhamento do que por sua familiaridade, como condição para a compreensão do fenômeno em estudo ${ }^{25,26}$.

$\mathrm{Na}$ condução do estudo, foram respeitados os aspectos éticos, conforme recomenda a resolução do Conselho Nacional de Saúde n. 466/2012. Por se tratar de pesquisa com dados documentais de caráter público, não houve a obrigatoriedade de submissão ao Comitê de Ética e Pesquisa.

\section{Resultados e discussão}

Do universo das novas escolas, 27 (51\%) são públicas e 26 (49\%), particulares. $\mathrm{Na}$ amostragem deste estudo encontramos $80 \%$ de públicas e $20 \%$ de privadas, sendo que os documentos de todas as públicas foram obtidos em sites institucionais, enquanto que somente em um terço das privadas isto pode ser verificado. Essa situação pode evidenciar que as escolas públicas favorecem o acesso a esses documentos, respeitando seu caráter público.

Todas as regiões do país estão contempladas no conjunto de escolas analisadas, estando distribuídas em 11 estados, sendo 12 públicas e três privadas, a maioria em cidades predominantemente de médio porte (até 200 mil habitantes). Destas, três características se destacam: a maioria se concentra no Nordeste, com forte perfil de interiorização, e são predominantemente instituiçôes federais.

Este perfil de distribuição geográfica e de administração das escolas é coerente com políticas públicas, como o Programa de Apoio a Planos de Reestruturação e Expansão das Universidades Federais (Reuni) e o Programa Mais Médicos. 
(2)

A seguir, são apresentados os dados que emanaram dos PPC, que seguem a lógica dos núcleos de sentido identificados, explorando cada uma de suas categorias de análise.

\section{Atenção à saúde}

Nesta dimensão, foram identificados três núcleos de sentido: processo saúdeadoecimento, produção de saúde e formação geral.

O processo saúde-doença está expresso nos PPC como um conceito que estrutura as práticas em saúde e transversaliza as ações educacionais. São duas as explicações assinaladas para esse processo. A pesar de não serem excludentes, a primeira explicação - multicausal - coloca no mesmo plano certas condições como alimentação; habitação; educação; renda; meio ambiente; trabalho; emprego; lazer; liberdade; acesso e posse da terra; e acesso a serviços de saúde. $\mathrm{Na}$ determinação social, a segunda explicação, o eixo explicativo do processo saúde-doença - refere-se aos diferentes modos de organização social da produção que geram diferenças significativas nos níveis de vida, a depender da posição social que os indivíduos e grupos ocupam ${ }^{6,927}$.

[...] elemento que deverá nortear o projeto pedagógico do curso, qual seja a adoção do conceito ampliado de saúde como referência para a sua concepção e de seu currículo. Por outras palavras, a despeito de a saúde comportar uma dimensão biológica fundamental, posto que alguma forma de desequilíbrio, em geral, reflete-se em patologias do corpo, ela não pode se resumir apenas a esta dimensão, já que fatores de outra natureza se convertem em determinantes do fenômeno saúde-doença. (E2)

[...] o conceito ampliado de saúde requer que sejam os futuros profissionais despertados para distintos aspectos da realidade em que se dá o fenômeno saúde-doença, de modo a subsidiá-los para uma leitura abrangente do mundo, da qual não escapa a política, entendida como o espaço de disputa e das relações de poder; a economia, como âmbito em que acontece a produção, circulação e distribuição de bens e serviços; a cultura, como repositório de todo o acervo de saberes do grupo [...]. E2

A determinação social do processo saúde-doença aparece de forma destacada nas DCN de 2014. Entretanto, essas diferenças não geraram desdobramentos na concepção curricular.

A produção do cuidado é outro núcleo de sentido no qual foram identificadas três categorias: clínica ampliada, paciente como sujeito e produção do cuidado em equipe.

Em relação ao modelo de clínica e ao processo de cuidado, as escolas apontam para a necessidade de superar o predomínio do biológico e da doença, indicando como alternativa o foco no sujeito que adoece e sofre inserido no seu contexto familiar e social. Associado a essa mudança, destaca-se nos documentos a adoção do conceito de clínica ampliada proposto por $\mathrm{Campos}^{28}$, que aponta outro entendimento de clínica, 
partindo do modelo biomédico para os aspectos subjetivos e sociais dos sujeitos e respeitando a característica singular de cada caso, visando estimular a autonomia e o protagonismo dos sujeitos.

Busca dialogar com as necessidades referidas pela pessoa sob seus cuidados ou responsável com as necessidades percebidas pelos profissionais de saúde, estimulando-a a refletir sobre seus problemas e a promover o autocuidado. Pactua as ações de cuidado, promovendo a participação de outros profissionais, sempre que necessário. (E2)

[...] incluir a perspectiva dos usuários, família e comunidade, favorecendo sua maior autonomia na decisão do plano terapêutico, respeitando seu processo de planejamento e de decisão considerando-se, ainda, os seus valores e crenças. (E5)

Em relação à produção do cuidado, os PPC demonstram que as escolas se preocupam com a inclusão de outros profissionais nessa dimensão da atenção, entendendo que uma equipe multiprofissional pode desenvolver uma atenção mais integral e resolutiva.

As escolas apontam principalmente duas dimensões do trabalho em equipe: interdisciplinaridade e multiprofissionalidade. Indicam que a prática interdisciplinar deve ser construída dia a dia na vivência do estudante, de modo que se torne uma atitude constante no seu cotidiano profissional e não uma atitude esporádica. Desta forma, os estudantes são expostos a situaçốes comuns de aprendizagem, pois frente a um mesmo problema, a complexidade da prática compartilhada por estudantes de diferentes áreas possibilita olhares diferentes, que proporcionam uma visão mais ampliada da realidade na saúde.

[...] a ênfase interdisciplinar favorece o redimensionamento das relaçôes entre diferentes conteúdos, contribuindo para que a fragmentação dos conhecimentos possa ser superada. Integrar também implica pensar em novas interações no trabalho em equipe multiprofissional, configurando trocas de experiências e saberes numa postura de respeito à diversidade, cooperação para efetivar práticas transformadoras, parcerias na construção de projetos e exercício permanente do diálogo. (E3)

Em relação à formação geral do médico, o discurso das escolas evidencia diferentes sentidos. Além do caráter técnico-humanista que está presente em todos, destaca-se como atribuição do médico generalista a oferta de ações de promoção, prevenção, recuperação e reabilitação da saúde, referindo-se à assistência integral; à resolubilidade em cerca de $85 \%$ dos problemas de saúde da população, que é coincidente com o que aponta a literatura ${ }^{11-13}$; e ainda à incorporação da lógica da universalidade no atendimento, ora representados pelos ciclos de vida, ora baseado nas especialidades médicas básicas. 
Demonstrar a capacidade de realizar consulta médica completa em atenção primária à saúde da criança, de gestantes, adultos e idosos de ambos os sexos. (E8)

Dominar conhecimentos referentes à atuação nas principais áreas médicas (Pediatria, Clínica Médica, Tocoginecologia, Cirurgia, Saúde Mental e Medicina Social). (E7)

Exercer cuidados integrais de saúde com resolubilidade esperada para a maior parte dos casos, identificando as situaçóes que precisam inserir-se nos sistemas de regulação para referência aos níveis especializados de atenção. E9

[...] a formação de um médico com proficiência de atuação profissional prioritariamente nos cenários de APS e U/E e integrada no Sistema de Saúde [...]. E3

Pode-se afirmar que a formação geral dos médicos é um campo polissêmico, persistindo ainda a ideia que a soma de conhecimentos das principais especialidades médicas é suficiente para preparar um profissional para cuidar de forma integral.

As DCN de $2014^{14}$ preveem a importância da formação médica em áreas estratégicas para o SUS, e as escolas se mostram alinhadas a essa política.

\section{Gestão em saúde}

Gestão em saúde é uma das áreas de competência presentes nas DCN de $2014^{14}$. Essa área nas DCN de 2001 tinha um caráter gerencial centrado na tomada de decisão, liderança, administração e gerenciamento. Nas DCN de 2014, a área de competência utiliza o conceito de gestão do cuidado. As escolas incorporam esse conceito e expressam nos seus PPC por meio dos termos "gestão da clínica" ${ }^{2}$ e "gestão do cuidado" 30,31.

Foram identificados na análise dos PPC três núcleos de sentido nesta dimensão: gestão do cuidado; competência gerencial; acompanhamento e avaliação do trabalho em saúde.

Todos os PPC pesquisados são unânimes em assumir a relação do cuidado com a gestão, ou seja, que o cuidado é passível de gestão e que deve ser incorporado nas respostas sanitárias a serem desenvolvidas.

Compreender os princípios da gestão da clínica e aplicá-los na organização da demanda. (E8)

Gestão do cuidado, com o uso de saberes e dispositivos de todas as densidades tecnológicas, de modo a promover a organização dos sistemas integrados de saúde para a formulação e desenvolvimento de planos terapêuticos individuais e coletivos. (E15) 
Gestão da clínica e gestão do cuidado, apesar de serem tratados como um mesmo tema, aparecem com sentidos diversos. O primeiro termo diz respeito à gestão como competência gerencial, que é apresentada no sentido do trabalho em equipe, desenvolvimento de liderança e gestão de conflitos; e o segundo está relacionado à gestão das ofertas de serviços do sistema de saúde, da organização das redes de atenção e da intersetorialidade, com vistas à integralidade do cuidado e à qualificação do itinerário/trajetória terapêutico do usuário ${ }^{30,31}$.

Os PPC abordam ainda o monitoramento clínico, principalmente como acompanhamento e avaliação do trabalho em saúde, incluindo a utilização de indicadores, protocolos, relação custo-benefício, custo-efetividade e auditoria, elementos que expressam a lógica do modelo de atenção gerenciada ${ }^{32}$.

Além desses sentidos, as escolas trazem alguns elementos que implicam na gestão com a eficácia do cuidado, melhores práticas e melhoria da assistência, consonantes com o conceito de governança clínica proposto por Gomes e colaboradores ${ }^{29}$.

\section{Educação em saúde}

Educação permanente como era designada nas DCN de 2001, foi substituída nas de 2014 pela seção Educação em Saúde, que é uma das grandes áreas de competência médica ${ }^{10,14}$.

A análise dos PPC permitiu identificar nesta dimensão quatro núcleos de sentido: abordagem pedagógica, estrutura curricular, compartilhamento de saberes e capacitação docente.

Historicamente, o processo ensino-aprendizagem tem se restringido à reprodução do conhecimento, no qual o docente assume um papel de transmissor de conteúdo, ao passo que ao discente cabe a retenção e repetição destes, de forma passiva e acrítica ${ }^{33-35}$.

Buscando superar estas características, há um movimento voltado à produção de mudanças com o uso de metodologias ativas de ensino-aprendizagem e à promoção de uma educação transformadora ${ }^{33-35}$.

As DCN de 2001 já apontavam para a necessidade de utilizar outras metodologias de ensino, e as DCN de 2014 vieram ratificar a importância desta mudança no modelo pedagógico adotado pelas escolas.

O número de escolas que tem adotado metodologias ativas é crescente no mundo e no Brasil ${ }^{36}$. Duas estratégias principais vêm sendo adotadas pelas escolas: a problematização e a aprendizagem baseada em problemas (ABP). Algumas escolas relatam utilizar tanto a $\mathrm{ABP}$ quanto a problematização, sendo que essas estratégias pedagógicas podem ser complementares. 
A aprendizagem baseada em problemas, na educação dos profissionais de saúde, tem três objetivos: a aquisição de um corpo integrado de conhecimentos, a aplicação de habilidades para resolver problemas e o desenvolvimento do raciocínio clínico. A problematização como metodologia de ensino, de estudo e de trabalho [...] integra a metodologia do curso como estratégia para estimular a curiosidade, assim como retoma o aprofundamento teórico a ser estudado [...]. (E2)

Uma escola cita a utilização da metodologia da espiral construtivista, que utiliza disparadores que simulam problemas da realidade.

Todas as escolas afirmam utilizar, em alguma medida, metodologias ativas de ensino-aprendizagem. Uma escola assume adotar um currículo híbrido.

[...] se trata de um modelo de currículo híbrido, onde possibilitamos a convivência de estratégias, fazeres e saberes ecléticos, que possuem como meta convergente a formação integral do indivíduo pautada numa aprendizagem significativa e problematizadora, que fará amplo uso de metodologias e dispositivos ativos de aprendizagem e ensino. (E7)

As escolas demonstram a relevância de se desenvolver atividades nos cenários reais dos serviços de saúde, nos quais é possível o contato com a realidade como ela se apresenta. Além disso, propóem a utilização de ambientes simulados, com diversas estratégias educacionais, como a utilização de simuladores, manequins e role-playing ${ }^{37}$.

Outro aspecto importante é a avaliação, que é considerada por alguns autores como a grande evidência do projeto pedagógico da escola ${ }^{16,38}$.

A avaliação formativa tem ganhado espaço, e as escolas estudadas valorizam este método avaliativo.

Avaliações formativas: serão realizadas de forma regular e ao longo do processo formativo, com a finalidade de se obter dados sobre o progresso dos alunos. Deste modo, a avaliação formativa poderá viabilizar oportunas correçôes dos desvios observados e reforçar as conquistas realizadas. (E6)

É sabido que nenhum método é capaz de isoladamente avaliar os múltiplos aspectos que envolvem a complexidade do saber médico; daí a necessidade da combinação dos métodos, como apresentado pelas escolas, tendo como foco a avaliação formativa ${ }^{39,40}$.

A análise dos PPC permite inferir que há um avanço em relação às estratégias educacionais adotadas pelos cursos, já que a maioria das escolas utilizam as aulas expositivas como complementares a outras estratégias alinhadas às metodologias ativas, como grupos tutoriais e team based learning (TBL).

As novas escolas médicas têm definido diversos arranjos para a sua organização curricular. Os trechos dos PPC registram predominantemente diretrizes para organização curricular nos moldes de currículo coleção ${ }^{41}$, com ciclos básico, clínico e internato 
médico. O currículo assim formatado constitui um fator que dificulta a integração das disciplinas, o que é fundamental para a interdisciplinaridade na formação, demonstrando dificuldade na sua superação.

[...] 2 anos compondo o primeiro ciclo de aprendizagem (integrando conhecimentos básicos aos aplicados e aos cenários de práticas relevantes, dando ênfase aos conhecimentos básicos), 2 anos no segundo ciclo de aprendizagem (ênfase nos conhecimentos aplicados) e 2 anos no terceiro ciclo de aprendizagem na modalidade Internato Médico. (E4)

Encontram-se também mençôes a currículos integrados ${ }^{41}$ nos PPC estudados. Esses conteúdos são apresentados de forma variada: módulos temáticos por ciclo vital, unidades por sistemas orgânicos ou atividades em torno de conceitos-chave.

As DCN de $2014^{14}$ determinam que deve-se inserir o aluno nos serviços de saúde desde as séries iniciais e ao longo de todo o curso, e as novas escolas acompanham essa recomendação. A aproximação dos estudantes com o cotidiano dos usuários e do serviço possibilita a produção de vínculos e tende a solidificar a formação na perspectiva da integralidade. Nos cenários de prática, os alunos desenvolvem vivências únicas, impossíveis de serem desenvolvidas em sala de aula.

As DCN de Medicina de 2014, tal como determina a Lei no 12.871, apontam que no mínimo 30\% da carga horária do internato seja desenvolvida na Atenção Básica e nos serviços de Urgência e Emergência, com predomínio da Atenção Básica, que deverá ser coordenada pela Medicina Geral de Família e Comunidade (MFC) ${ }^{14}$. Observa-se nos PPC que as escolas seguem essa recomendação. Dessa forma, o egresso de Medicina deve estar apto para atuar em todos os pontos da rede de atenção, principalmente nas suas portas de entrada.

$\mathrm{Na}$ categoria "Relação ensino-serviço", emergiram dos PPC duas subcategorias: horizontalidade nas relações e Contrato Organizativo de Ação Pública Ensino-Serviço (Coapes). O estabelecimento do Coapes é uma inovação das DCN de 2014 em relação às de $2001 \mathrm{e}$, embora seja um dispositivo recente, as escolas relatam sua utilização.

Em relação ao compartilhamento de saberes, no âmbito da educação com a equipe e para o trabalho, observa-se que o conceito de educação permanente em saúde não é incorporado pelas escolas, que os confunde com educação continuada.

[...] ter curiosidade técnico-científica e realizar educação permanente (busca constante de informaçóes e autoaprendizado). (E5)

Atualizar-se permanentemente na sua vida profissional, participando constantemente de programas de educação continuada. (E7)

Ainda hoje, a denominada "educação bancária” tem sido uma realidade nas práticas de saúde, reproduzindo o discurso biologicista e intervençôes normalizadoras, em uma relação assimétrica entre educador e educando, ou entre profissionais e usuários ${ }^{33-35,38}$. 
(e)

Alguns PPC apontam que a educação popular em saúde deve ser uma estratégia vivenciada pelos alunos, visando ao estímulo à autonomia dos sujeitos ${ }^{42}$.

As DCN de Medicina de $2014^{14}$ determinam que os cursos deverão manter permanente programa de formação e desenvolvimento da docência em saúde. Todas as escolas analisadas demonstram estruturação de programas de capacitação docente, algumas na lógica da educação permanente e outras na da educação continuada.

Esses achados encontram proximidade com outros estudos ${ }^{19,43,44}$ que apontam também para a inclusão dos princípios das DCN nos PPC e currículos das escolas médicas. Neles, a incorporação desses princípios aparece com maior ou menor expressão. Alguns apontam a ausência do método clínico centrado na pessoa e nas decisóes compartilhadas; outros, diferenças entre a metodologia de ensino e concepção do curso.

\section{Considerações finais}

Reconhece-se que o contexto institucional com a criação do Programa Mais Médicos em 2013 e a elaboração das DCN de Medicina em 2014 estimularam o aumento de ofertas de vagas para formação de médicos. Essa política pública estabelece que os cursos sejam concebidos a partir de novos paradigmas educacionais e voltados para atuação no SUS, em especial, nas equipes de Saúde da Família.

Esse delineamento político abriu uma oportunidade para as IES. Pode-se afirmar que a capacidade indutora dessas normativas institucionais foi muito efetiva, sendo criadas, no período deste estudo, 53 escolas por IES públicas e privadas, com componentes do fenômeno da interiorização. Para serem aprovadas, apresentaram ao Ministério da Educação seus PPC alinhados ao conjunto de regras e normas definidas nesses documentos legais.

A análise dos PPC das IES que foram objeto deste estudo propiciou a identificação de elementos que apontam para uma grande convergência com as mudanças pretendidas pela DCN 2014. Há um claro direcionamento para o modelo da clínica ampliada, demonstrando alinhamento com o modelo de atenção integral adotado pelas DCN de Medicina de 2014.

As escolas procuram adotar a atenção à saúde na perspectiva do cuidado, o que evidencia uma superação do modelo biologicista adotado historicamente.

Há ainda divergências sobre a concepção de formação geral do médico. Dessa forma, essa questão, essencial para a estruturação dos currículos, permanece como um grande desafio a ser superado pelas escolas. Estruturar a formação a partir da MFC pode ser uma diretriz norteadora para a formação de médicos generalistas.

A formação voltada para a Atenção Básica pode ser considerada uma conquista, pois prevê um cuidado integral, humanizado, interprofissional e que atenda às reais necessidades da população.

Outra conquista importante das DCN de 2014 foi trazer o conceito de gestão do cuidado, e as escolas acompanham esse avanço, demonstrando que compreendem que o cuidado é passível de gestão. Vale destacar que os PPC apresentam mais elementos 
e estratégias para o desenvolvimento da competência atenção à saúde do que para Gestão em Saúde, o que pode denotar uma dificuldade da incorporação de competências gerenciais pelo médico.

Em relação à educação em saúde, verificou-se uma ampla adesão às metodologias ativas de ensino-aprendizagem, mostrando forte alinhamento às DCN de 2014. Entretanto, ao se analisar a matriz curricular e as estratégias educacionais, verifica-se na prática currículos híbridos ou tradicionais. Isso pode indicar uma certa resistência a mudanças conceituais do currículo, ou ainda que as escolas passam por um momento de transição.

As escolas ainda estruturam seus currículos em ciclos, formato que está muito relacionado ao modelo flexneriano e que se esperava já ter sido superado. Assumem também a importância da integração curricular, porém, esta se dá predominantemente entre as disciplinas do mesmo ciclo, e não entre os ciclos básico, clínico e internato. Dessa forma, outro grande desafio a ser superado pelas escolas é a articulação entre o currículo proposto e o prescrito, pois nos PPC estão expressos os elementos centrais das DCN, mas quando se analisa o currículo prescrito, há uma lacuna na estrutura dos cursos, principalmente na organização curricular, o que pode sugerir a manutenção de currículo coleção.

Ao estruturar programa de formação e desenvolvimento da docência, as escolas demonstram um compromisso com a mudança na formação profissional do médico.

Analisando atentamente os PPC, observa-se que existe um deslocamento do paradigma flexneriano para o da integralidade, embora ainda com algumas fragilidades.

Como este estudo foi realizado com o currículo formal, uma das suas limitações é não retratar outras dimensóes, como o currículo paralelo e o currículo oculto. Nesse sentido, recomenda-se que sejam realizadas novas pesquisas a fim de incluir a visão de docentes e discentes sobre o currículo vivido e seu impacto nas mudanças almejadas para a formação do médico. 


\section{Contribuições dos autores}

Todos os autores participaram ativamente de todas as etapas de elaboração do manuscrito.

\section{Conflito de interesse}

Os autores não têm conflito de interesse a declarar.

\section{Direitos autorais}

Este artigo está licenciado sob a Licença Internacional Creative Commons 4.0, tipo BY (https://creativecommons.org/licenses/by/4.0/deed.pt_BR).

\section{(cc) BY}

\section{Editora}

Vera Lúcia Garcia

Editora associada

Rosana Rossit

Submetido em

$04 / 10 / 20$

Aprovado em

$28 / 12 / 20$

\section{Referências}

1. Abdalla IG, Stella RCR, Perim GL, Aguiar-da-Silva RH, Lampert JB, Costa NMSC. Projeto Pedagógico e as mudanças na educação médica. Rev Bras Educ Med. 2009; 33(1):44-52.

2. Neto AFP. Tendências de mudanças na formação médica no Brasil: tipologia das escolas. Cienc Saude Colet. 2002; 7(4):935-40.

3. Feuerwerker LCM. Micropolítica e saúde: produção do cuidado, gestão e formação. Porto Alegre: Rede UNIDA; 2014.

4. Gomes R, Francisco AM, Tonhom SFR, Costa MCG, Hamamoto CG, Pinheiro OL, et al. A formação médica ancorada na aprendizagem baseada em problema: uma avaliação qualitativa. Interface (Botucatu). 2009; 13(28):71-83.

5. Almeida MJ. A educação médica e as atuais propostas de mudança: alguns antecedentes históricos. Rev Bras Educ Med. 2001; 25(2):42-52.

6. Pinheiro R, Mattos RA. Os sentidos da integralidade. Rio de Janeiro: UERJ, ABRASCO; 2006.

7. Lampert JB, Perim GL, Aguilar-da-Silva RH, Stella RCDR, Abdala IG, Costa NMDSC. Mundo do trabalho no contexto da formação médica. Rev Bras Educ Med. 2009; 33(1):35-43. 
8. Beltrame RL. A formação do médico: um debate à luz das diretrizes curriculares nacionais [tese]. São Paulo: Pontíficia Universidade Católica de São Paulo; 2006.

9. Silva FA. Processo autoavaliativo do desenvolvimento docente em escolas médicas brasileiras: espelhos e reflexos nas percepções dos atores institucionais [tese]. Goiânia: Universidade Federal de Goiás; 2017.

10. Brasil. Ministério da Saúde. Promed: Programa de incentivo a mudanças curriculares nos cursos de medicina. Brasília: Ministério da Saúde; 2005.

11. Brasil. Ministério da Saúde. Ministério da Educação. Pró-Saúde: programa nacional de reorientação da formação profissional em saúde: objetivos, implementação e desenvolvimento potencial. Brasília: Ministério da Saúde; 2007.

12. Andrade MRS. Formação em saúde: experiências e pesquisas nos cenários de prática, orientação teórica e pedagógica. Blumenau: Edifurb; 2011.

13. Brasil. Ministério da Saúde. Secretaria de Gestão do Trabalho e da Educação na Saúde. Mais médicos - dois anos: mais saúde para os brasileiros. Brasília: Ministério da Saúde; 2015.

14. Brasil. Ministério da Saúde. Diretrizes curriculares nacionais do curso de graduação em medicina. Brasília: Ministério da Saúde; 2014.

15. Oliveira FP, Pinto HA, Figueiredo AM, Cyrino EG, Oliveira Neto AV, Rocha VXM. Programa mais médicos: avaliando a implantação do eixo formação de 2013 a 2015. Interface (Botucatu). 2019; 23 Supl 1:e170949.

16. Sordi MRL, Mendes GSCV, Cyrino EG, Alexandre FLF, Manoel CM, Lopes CVM. Experiência de construção coletiva de instrumento autoavaliativo a serviço da formação médica referenciada nas Diretrizes Curriculares Nacionais (DCN) pautadas no Programa Mais Médicos. Interface (Botucatu). 2020; 24:e190527.

17. Furlanetto DLC, Bastos MM, Silva Júnior JW, Pinho DLMl. Reflexôes sobre as bases conceituais das diretrizes curriculares nacionais em cursos de graduação em saúde. Comun Cienc Saude. 2014; 25(2):193-202.

18. Rezende VLM, Rocha BS, Naghettini A, Fernandes MR, Pereira ERS. Percepção discente e docente sobre o desenvolvimento curricular na atenção primária após Diretrizes Curriculares de 2014. Rev Bras Educ Med. 2019; 43(3):91-9.

19. Cândido PTS, Batista NA. O internato médico após as diretrizes curriculares nacionais de 2014: um estudo em escolas médicas do estado do Rio de Janeiro. Rev Bras Educ Med. 2019; 43(3):36-45.

20. Vieira SP, Pierantoni CR, Magnago C, Ney MS, Miranda RG. A graduação em medicina no Brasil ante os desafios da formação para a atenção primária à saúde. Saude Debate. 2018; 42(esp 1):189-207.

21. Ferreira MJM, Ribeiro KG, Almeida MM, Sousa MS, Ribeiro MTAM, Machado MMT, et al. Novas diretrizes curriculares nacionais para os cursos de medicina: oportunidades para ressignificar a formação. Interface (Botucatu). 2019; 23 Supl 1:e170920.

22. Rocha VXM. Reformas na educação médica no Brasil: estudo comparativo entre as diretrizes curriculares nacionais dos cursos de graduação em medicina de 2001 e 2014 [dissertação]. Santos: Universidade Católica de Santos; 2017.

23. Minayo MCS. O desafio do conhecimento: pesquisa qualitativa em saúde. São Paulo: Hucitec; 2014.

24. Bardin L. Análise de conteúdo. São Paulo: Ediçóes 70; 2011.

25. Gadamer HG. Verdade e método. Petrópolis: Vozes; 2012. 
26. Ayres JRCM. Hermenêutica e humanização das práticas de saúde. Cienc Saude Colet. 2005; 10(3):549-60.

27. Buss PM, Pellegrini Filho A. A Saúde e seus determinantes sociais. Physis. 2006; 17(1):77-93.

28. Campos GWDS, Amaral MA. A clínica ampliada e compartilhada, a gestão democrática e redes de atenção como referenciais teórico-operacionais para a reforma do hospital. Cienc Saude Colet. 2007; 12(4):849-59.

29. Gomes R, Lima VV, Oliveira JM, Schiesari LMC, Soeiro E, Damázio LF, et al. A polissemia da governança clínica: uma revisão da literatura. Cienc Saude Colet. 2015; 20(8):2431-9.

30. Santos AM, Giovanella L. Gestão do cuidado integral em saúde: estudo de caso em região de saúde da Bahia, Brasil. Cad Saude Publica. 2016; 32(3):e00172214.

31. Grabois, V. Gestão do cuidado. In: Oliveira RG, Grabois V, Mendes Junior WV, organizadores. Qualificação de gestores do SUS. Rio de Janeiro: EAD/Ensp; 2009. p. 159-97.

32. Paim JS. Modelos de atenção à saúde no Brasil. In: Giovanella L, Escorel S, Lobato LVC, Noronha JC, Carvalho AI, organizadores. Políticas e sistemas de saúde no Brasil. Rio de Janeiro: Editora Fiocruz/Centro Brasileiro de Estudos de Saúde; 2012. p. 547-73.

33. Mitre SM, Siqueira-Batista R, Girardi-de-Mendonça JM, Morais-Pinto NM, Meirelles $\mathrm{CDAB}$, Pinto-Porto $\mathrm{C}$, et al. Metodologias ativas de ensino-aprendizagem na formação profissional em saúde: debates atuais. Cienc Saude Colet. 2008; 13(2):2133-44.

34. Diesel A, Baldez ALS, Martins SN. Os princípios das metodologias ativas de ensino: uma abordagem teórica. Thema. 2017; 14(1):268-88.

35. Lima VV. Espiral construtivista: uma metodologia ativa de ensino-aprendizagem. Interface (Botucatu). 2017; 21(61):421-34.

36. Gontijo ED, Alvim CG, Lima MECC. Manual de avaliação da aprendizagem no curso de graduação em medicina. Rev Docencia Ensino Super (Belo Horizonte). 2015; 5(1):205-326.

37. Rabelo L, Garcia VL. Role-play para o desenvolvimento de habilidades de comunicação e relacionais. Rev Bras Educ Med. 2015; 39(4):586-96.

38. Sordi MRL. Problematizando o papel da avaliação da aprendizagem nas metodologias inovadoras na área da saúde. Rev Educ PUC-Campinas. 2000; (9):52-61.

39. Royal College of General Practitioners. Think general pratictioner. London: NHS; 2016.

40. World Health Organization Europe. Framework for professional and administrative development of general practice/family [Internet]. Copenhagen: WHO; 1998 [citado 13 Nov 2020]. Disponível em: https://apps.who.int/iris/bitstream/ handle/10665/108066/E58474.pdf?sequence=1\&isAllowed=y

41. Berstein B. Pedagogy, symbolic control, and identity: theory, research, critique (critical perspectives on literacy and education). London: Taylor \& Francis; 1996.

42. Monteiro EMLM. Educação popular em saúde. Recife: UFPE; 2015.

43. Oliveira C, Senger MH, Ezequiel OS, Amaral E. Alinhamento de diferentes projetos pedagógicos de cursos de medicina com as diretrizes curriculares nacionais. Rev Bras Educ Med. 2019; 43(2):143-51.

44. Rezende VLM, Rocha BS, Naghettini AV, Pereira ERS. Análise documental do projeto pedagógico de um curso de medicina e o ensino na atenção primária à saúde. Interface (Botucatu). 2019; 23 Supl 1:e170896. 
The Brazilian national curricular guidelines on health education steer curriculum composition so as to organize professional training in this field. Pedagogical projects for courses connect these guidelines to pedagogical practices. This study assessed the repercussions of the 2014 curricular guidelines for the pedagogical projects of medical schools that were created after publication of the guidelines. A qualitative approach was taken, with analysis on documents of these projects guided by critical hermeneutics. Strong alignment between these projects and the curricular guidelines was found. A shift from the Flexner paradigm to comprehensiveness was identified, such that the professional training tended towards the real needs of the population. Differences in the conception of general medical practitioners and in the use of active teaching-learning methodologies were found. Curricular conceptions in which the proposed teaching-service-community integration did not seem to be guaranteed were evident, thus suggesting that proposals based on disciplines had remained present.

Keywords: Medical education. Curriculum. Undergraduate medical education. Higher education policy.

Las Directrices Curriculares Nacionales orientan la constitución de los currículos para ordenamiento de la formación profesional en salud y los Proyectos Pedagógicos de los Cursos las articulan con las prácticas pedagógicas. Esta investigación analizó las repercusiones de las Directrices Curriculares de 2014 en los Proyectos Pedagógicos de las escuelas médicas creadas después de su publicación. De abordaje cualitativo, hace análisis documental de los referidos proyectos orientados por la hermenéutica crítica. Los hallazgos revelan un fuerte alineamiento de los proyectos analizados con las directrices curriculares. Se identificó un desplazamiento del paradigma flexneriano para la integralidad, tendiendo a la formación de profesionales enfocada en las necesidades reales de la población. Otros hallazgos son las diferencias en la concepción del médico de formación general y en la utilización de las metodologías activas de enseñanza aprendizaje. Se evidenciaron también matrices curriculares con concepciones cuya propuesta de integración enseñanza-servicio-comunidad no parecía estar garantizada, sugiriendo el mantenimiento de propuestas con base en asignaturas.

Palabras clave: Educación médica. Currículo. Educación de graduación en medicina. Política de educación superior. 